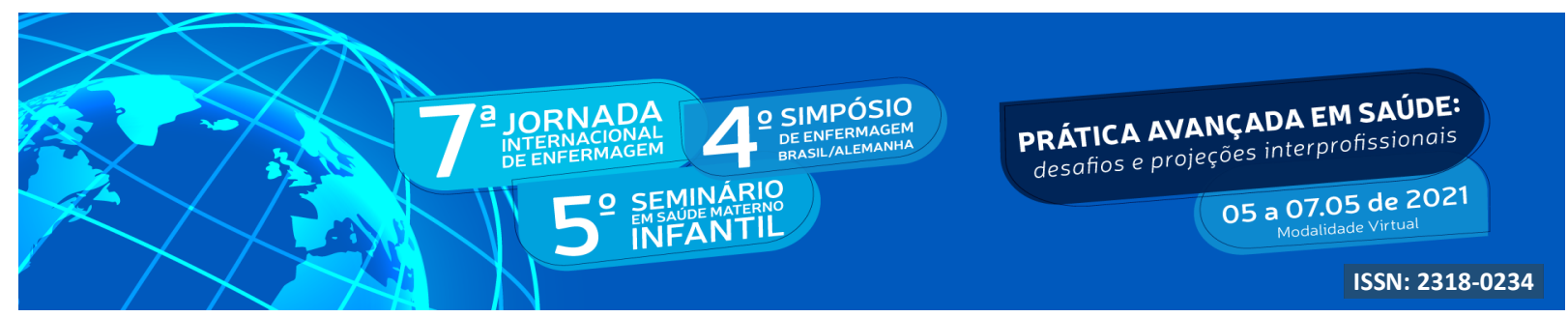

DOI: http://doi.org/10.48195/jie2021-057

\title{
GUIÃO DE ENTREVISTA SEGUNDO O MODELO TEÓRICO DO AJUSTE DOS ADOLESCENTES AO CANCRO PARENTAL ${ }^{1}$
}

\author{
Ana Filipa Sousa²; Margarida Reis Santos ${ }^{3}$; Lurdes Lomba ${ }^{4}$
}

\begin{abstract}
RESUMO
O cancro parental apresenta impacto na díade pais-filhos. Os adolescentes são mais vulneráveis que as crianças a esta situação, correndo risco de desenvolverem problemas psicológicos, comportamentais e psicossociais. A intervenção de enfermagem na díade que vivencia o cancro parental é essencial para ajudar estas famílias a enfrentarem a situação.

Este estudo objetiva apresentar um guião de entrevista para adolescentes que vivenciam o cancro parental, à luz do modelo teórico do ajuste dos adolescentes ao cancro parental.

A entrevista semiestruturada pretende avaliar os fatores que contribuem para o ajuste dos adolescentes nomeadamente as variáveis moderadoras e mediadoras. As intervenções de enfermagem promotoras do ajuste devem ter por base todas ou algumas das variáveis mediadoras.

Os resultados permitirão avaliar as necessidades dos adolescentes e conjuntamente com os resultados de uma scoping review realizada, servirão de base para a construção de um programa de intervenção de enfermagem.
\end{abstract}

Palavras-chave: Adolescentes; Cancro Parental; Enfermagem; Modelo Teórico.

\begin{abstract}
Parental cancer has an impact on the parent-child relation. Adolescents are more vulnerable than children to this situation, because of the risk of developing psychological, behavioral and psychosocial problems. Nursing intervention in the family that experiences parental cancer is essential to help them to face these situation.

This study aims to present an interview guide for adolescents who experience parental cancer, in the light of the theoretical model of the adjustment of adolescents to parental cancer.

The semi-structured interview aims to assess the factors that contribute to the adjustment of adolescents, like the moderating and mediating variables. Nursing interventions that promote adjustment must be based on all or some of the mediating variables.

The results will allow assessing the needs of adolescents and, together with the results of a scoping review carried out, will serve as a basis for the construction of a nursing intervention program.

Key Words: Adolescents; Nursing; Parental Cancer; Theorical Model.

\footnotetext{
${ }^{1}$ Trabalho de investigação no âmbito do doutoramento em Ciências de Enfermagem.

${ }^{2}$ Mestre em Enfermagem de Saúde Infantil e Pediatria. Enfermeira Especialista no IPO de Coimbra. Doutoranda em Ciências de Enfermagem no ICBAS - UP (UICISA:E). E-mail: afilipas87@gmail.com

${ }^{3}$ Orientador. Doutora em Ciências de Enfermagem. Professora Coordenadora. ESEP (CINTESIS). E-mail: mrs@esenf.pt

${ }^{4}$ Orientador. Doutora em Ciências de Enfermagem. Professora Adjunta. ESEnfC (UICISA: E). E-mail: mlomba@esenfc.pt
} 


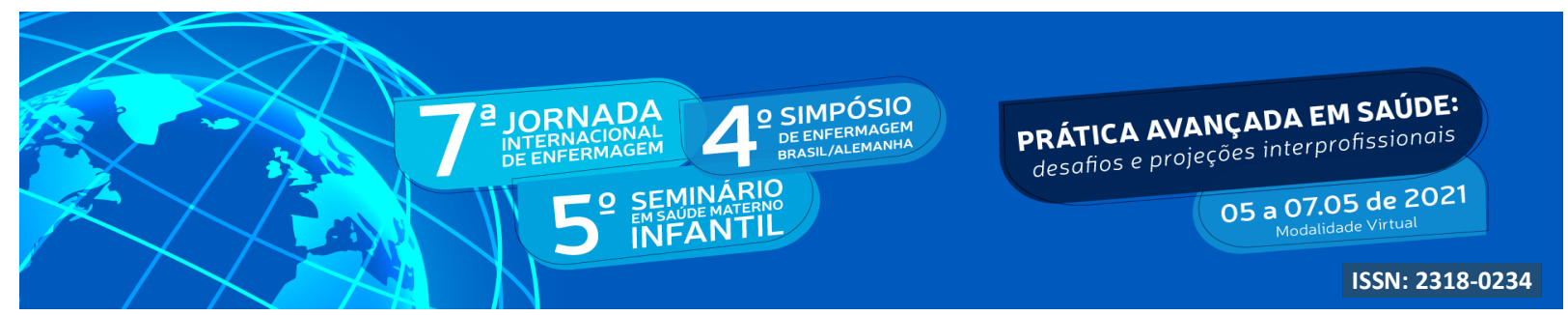

\section{INTRODUÇÃO}

A incidência de cancro nas faixas etárias mais jovens, tem aumentado consideravelmente nas últimas décadas. Estima-se que a nível internacional 15\% das pessoas com cancro têm entre 20 e 50 anos (O'Neill, O’Neill, \& Semple, 2019). Embora, na população portuguesa não seja conhecida a taxa de incidência de cancro nesta faixa etária, o diagnóstico é cada vez mais comum nos adultos jovens. As implicações do cancro em pessoas desta faixa etária, são diferentes das apresentadas pelos indivíduos mais velhos, pois muitos ainda trabalham ou estudam e têm filhos menores de 18 anos (Alexander, O'Connor, Rees, \& Halkett, 2019).

O cancro parental compreende a vivência da doença oncológica, numa idade precoce da vida adulta em pessoas com filhos dependentes (Barbosa, 2015), representando um fator de stress significativo não só para os doentes, devido às exigências da doença e dos tratamentos inerentes, muitas vezes incompatíveis com o cuidar de um filho (SPO, 2007), como também para os filhos, devido à potencial ameaça de morte dos pais, acarretando alterações na interação da díade (Su \& Ryan-Wenger, 2007).

Nos Estados Unidos da América, em 2019, mais de 380.000 crianças/adolescentes, foram confrontadas com o diagnóstico de cancro num progenitor (Lewis, Zahlis, \& Shands, 2020). Em Portugal não são conhecidos dados estatísticos referentes ao número de casos de crianças e adolescentes cujos pais têm cancro, no entanto reconhece-se que o impacto do diagnóstico de cancro nos pais, acarreta sofrimento e alterações na vida das crianças e adolescentes que vivenciam este processo.

Os filhos dos doentes oncológicos apresentam risco aumentado de sofrerem perturbações de desenvolvimento (Alexander et al., 2019), problemas psicológicos, emocionais, comportamentais (Shah, Armaly, \& Swieter, 2017) e psicossociais, incluindo ansiedade, isolamento, tristeza, raiva, depressão, dificuldades escolares/lúdicas, alterações nas dinâmicas familiares e stress pós-traumático (Ellis, Wakefield, Antill, Burns, \& Patterson, 2016).

Os adolescentes são considerados mais vulneráveis que as crianças, quando 


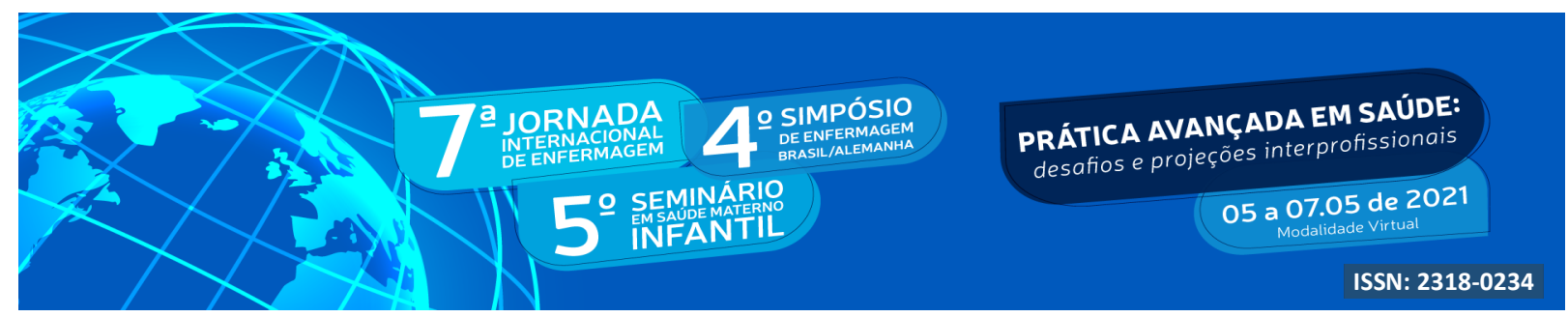

confrontados com a doença oncológica dos pais, demonstrando elevados níveis de ansiedade e depressão (Azarbarzin, Malekian, \& Taleghani, 2015; Grabiak, Bender, \& Puskar, 2007; Phillips, 2014). A aptidão para o pensamento abstrato e o aumento das habilidades cognitivas, torna-os mais suscetíveis à angústia, porque detêm maior conhecimento sobre as possíveis consequências, estando mais despertos para as perdas e dor física e emocional dos pais. Nesta faixa etária, é normal que se preocupem com o impacto da doença dos pais nas suas atividades diárias, nomeadamente na escola, desporto/lazer e, também, no seu relacionamento com os amigos. Por este motivo, podem apresentar mudanças no desempenho escolar, queixas físicas de dor, desconforto e alterações sociais e interpessoais (Azarbarzin et al., 2015). Resultante do normal processo de transição inerente ao crescimento/desenvolvimento, os adolescentes que vivenciam a experiência de cancro parental vivem sob pressão constante, na medida em que lutam pela independência e concomitantemente confrontam-se com a necessidade de apoiar os pais, tanto a nível físico como emocional (Azarbarzin et al., 2015).

Decorrente do desenvolvimento da identidade (Opperman \& Cassandra, 2001), alguns podem querer ser envolvidos na tomada de decisão sobre os tratamentos dos pais. Os adolescentes podem, também, apresentar preocupações relacionadas com a aparência dos pais e com as implicações que esse facto pode ter na sua relação com os outros, nomeadamente os pares.

Entre as consequências que o cancro parental acarreta nos padrões da vida familiar dos adolescentes, destaca-se a inversão de papéis, que pode ser difícil de encarar, tanto para os pais como para os adolescentes (Grabiak, et al., 2007; SPO, 2007). Considerando os papéis como comportamentos sociais característicos e esperados pela sociedade em determinada fase de vida, os adolescentes são capazes de aceitar e assumir responsabilidades domésticas que os pais não conseguem realizar por se encontrarem doentes, incluindo o cuidar dos irmãos mais novos (Grabiak, et al., 2007). Estas alterações nas dinâmicas familiares podem afetar a qualidade de vida dos adolescentes e prejudicar o seu envolvimento nas atividades escolares e extracurriculares com o grupo de pares (Phillips, 2014).

Considerando as tendências demográficas e as consequências que o cancro parental acarreta para a família, atendendo ao desafio único desta vivência, não só para os doentes como para os filhos, esta problemática enfatiza a necessidade da intervenção de enfermagem (Tamura, Yamazaki, \& Uchibori, 2021). Contudo, e apesar da pertinência do fenómeno e do 


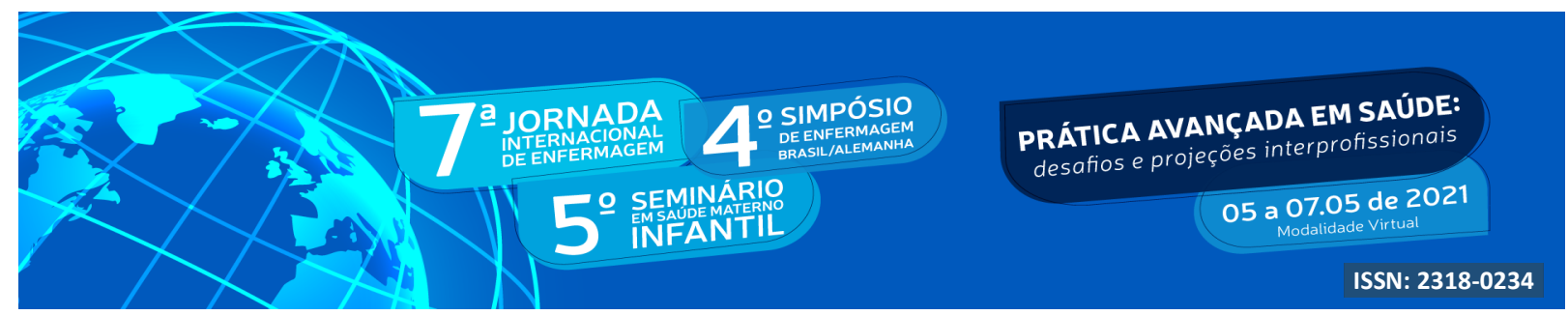

real impacto na díade pais-filhos, não existem orientações específicas para os profissionais de saúde relativas às famílias que vivenciam esta situação (Alexander et al., 2019; Arber \& Odelius, 2017).

A criação e a implementação de programas de intervenção para adolescentes e pais que vivenciam o cancro parental, requer um planeamento rigoroso (Inhestern, Haller, Wlodarczyk, \& Bergelt, 2016), para serem eficazes no desenvolvimento das habilidades parentais (Lewis, Zahlis, \& Shands, 2020), e produzirem resultados positivos nos adolescentes (Ellis et al., 2016), nomeadamente a nível da compreensão sobre o cancro dos pais, desenvolvimento de mecanismos de coping, melhoria do humor e comportamento adequado (Inhestern et al., 2016).

Neste contexto, considera-se premente a intervenção do enfermeiro em adolescentes e pais que vivenciam o cancro parental, recorrendo a um modelo teórico como padrão de referência para a definição de intervenções de enfermagem autónomas que respondam às necessidades da díade, promovendo-se a “(...) adaptação às mudanças na saúde e dinâmica familiar" (OE, 2010, p. 1).

Como base conceptual para a intervenção de enfermagem para adolescentes e pais que vivenciam o cancro parental, considerou-se como referência o modelo teórico do ajuste dos adolescentes ao cancro parental, proposto por Su \& Ryan-Wenger (2007).

O modelo apresenta como princípios filosóficos, a teoria do desenvolvimento infantil de Piaget (características do desenvolvimento do adolescente em termos cognitivos, emocionais e sociais), a teoria do Stress e Coping de Lazarus \& Folkman, e a teoria dos Sistemas Familiares de Bowen. Neste sentido, Su \& Ryan-Wenger (2007), consideram que o modelo ilustra a forma como conceitos importantes como ajuste e adaptação, se relacionam e como algumas variáveis moderam ou medeiam os resultados.

O conceito de ajuste foi considerado pelos autores como uma resposta a curto prazo do adolescente, enquanto a adaptação reporta-se às consequências a longo prazo no adolescente, decorrentes da vivência do cancro parental.

A conceção do modelo baseia-se na análise de programas de intervenção para famílias que vivenciaram o cancro parental e assenta no princípio de que o diagnóstico de cancro, invariavelmente conduz a stress psicológico e social nos adolescentes. Os fatores que 


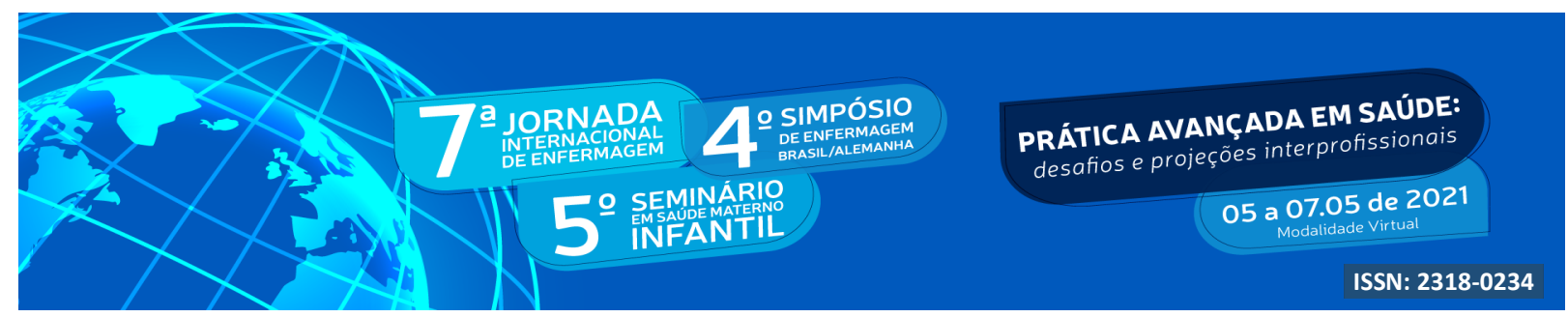

contribuem para o ajuste dos adolescentes podem ser moderadores e mediadores. As intervenções de enfermagem têm por base todas ou algumas das variáveis mediadoras. As variáveis são influenciadas pela intervenção e o resultado é a resposta à interação das variáveis moderadoras, mediadoras e intervenções de enfermagem. Como resultado, pode verificar-se um bom ou mau ajuste dos adolescentes ( $\mathrm{Su} \&$ Ryan-Wenger, 2007). As intervenções de enfermagem que considerem o impacto de variáveis moderadoras e mediadoras, ou ambas, promovem o ajuste.

A figura 1 representa esquematicamente o modelo teórico do ajuste do adolescente ao cancro parental, proposto por Su \& Ryan-Wenger (2007).

Figura 1: Ajuste do adolescente ao cancro parental.

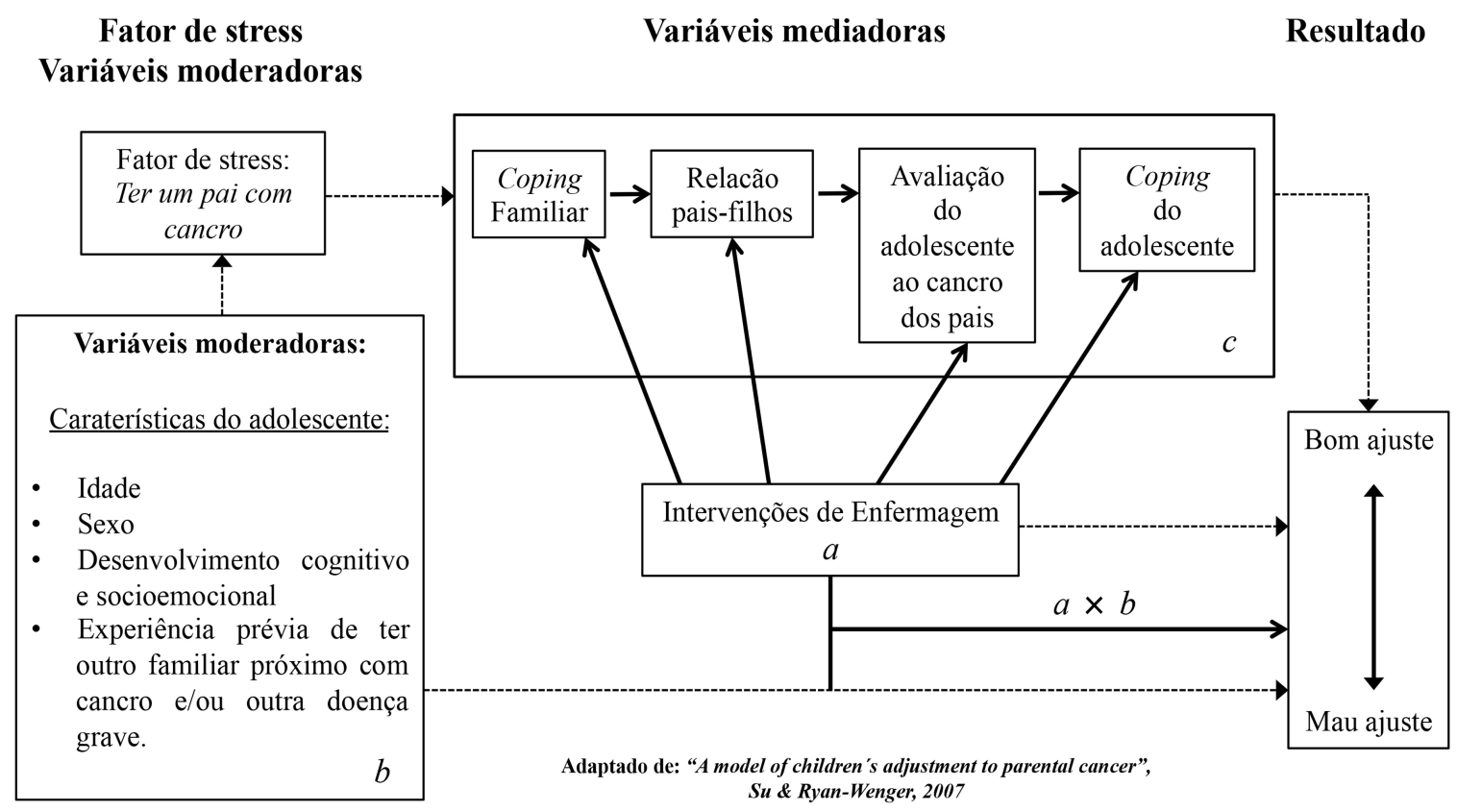

A variável mediadora coping familiar compreende todos os esforços cognitivos e comportamentais dos pais para gerirem, reduzirem ou tolerarem as necessidades internas e/ou externas da família que resultam do diagnóstico de cancro parental. A variável mediadora relação pai-filho, engloba a disponibilidade física e emocional dos pais para com o adolescente, incluindo a comunicação pais/filhos (padrão e frequência de conversas) e a 


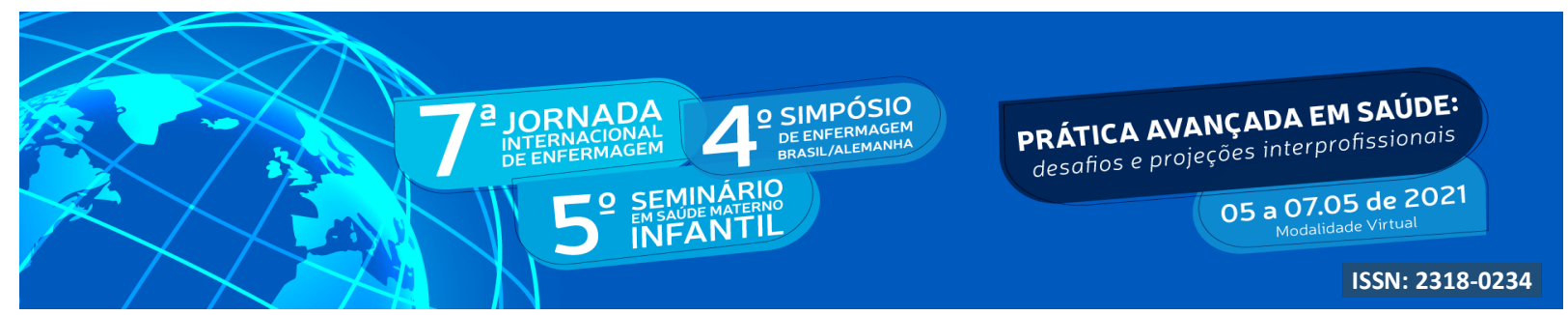

perceção do adolescente quanto ao apoio parental no que se refere aos seus problemas pessoais. A variável mediadora avaliação do adolescente sobre o cancro da mãe/do pai, referese ao julgamento do adolescente sobre a doença do progenitor. Pode ser classificada como: "Irrelevante" (sem interferência no bem-estar do adolescente); "Positivo" (o cancro parental não excede os recursos do adolescente e as consequências da vivência da doença parental são positivas); e "Stressante". Cada situação stressante pode ser definida em "Dano/perda", "Ameaça" ou "Desafio". A avaliação de "Dano/perda" corresponde a lesão ou danos ocorridos, como por exemplo, a perda de autoestima ou a deterioração da relação pais-filho. Já uma situação avaliada como "Ameaça", refere-se a um potencial de dano ou perda, enquanto "Desafio" é uma situação que se apresenta como uma oportunidade de crescimento, domínio ou ganho para o adolescente (Su \& Ryan-Wenger, 2007). A variável mediadora coping do adolescente, reporta-se aos esforços cognitivos e comportamentais do adolescente para gerir, reduzir ou tolerar as necessidades internas e/ou externas da família que advêm do diagnóstico de cancro de um dos pais. $\mathrm{O}$ adolescente pode ainda apresentar coping focado no problema: ajustando-se ou mudando o ambiente; coping focado na emoção: normalizando as emoções relacionadas com o fator de stress (cancro parental); ou estratégias de procura de apoio social, causado pelo cancro de um dos pais (Su \& Ryan-Wenger, 2007).

Segundo os autores, o modelo revela um esforço para resumir a teoria e a pesquisa sobre os efeitos do cancro parental nos adolescentes, podendo ser utilizado para orientar os profissionais de saúde a desenvolverem programas dirigidos a famílias afetadas pelo cancro (Su \& Ryan-Wenger, 2007).

\section{OBJETIVO}

Neste trabalho objetiva-se apresentar um guião de entrevista para adolescentes que vivenciam o cancro parental, segundo o modelo "Children's Adjustment to Parental Cancer: A Theorical Model Development”, de Su \& Ryan-Wenger (2007). 


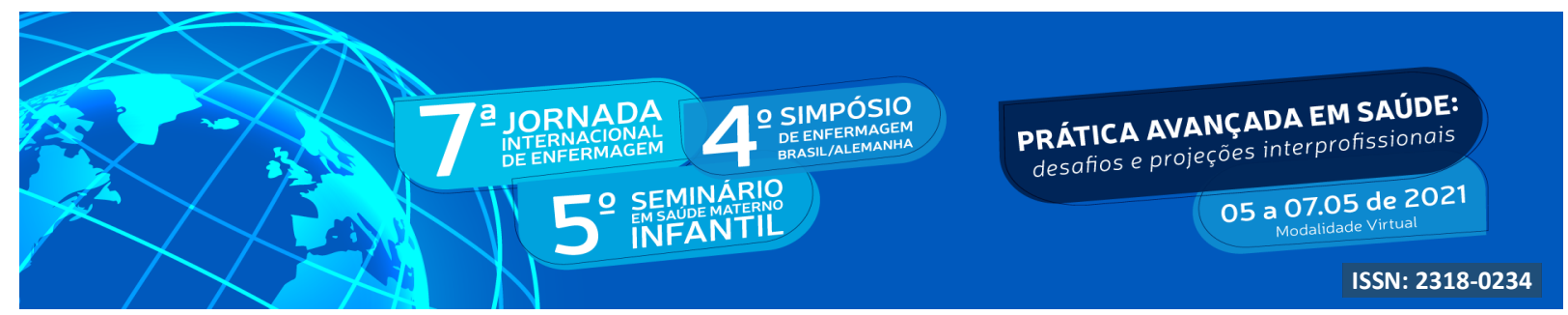

\section{METODOLOGIA}

Para sistematizar a entrevista ao adolescente que vivencia o cancro parental, foi elaborado um guião de entrevista semiestruturada, alicerçado nos princípios metodológicos e conceitos apresentados no modelo conceptual do ajuste dos adolescentes ao cancro parental de Su \& Ryan-Wenger (2007). Previamente, foi realizada uma pesquisa sobre a evidência produzida acerca do modelo teórico, seguindo-se a elaboração do guião de entrevista semiestruturada e posterior discussão com dois investigadores experts na área.

O guião de entrevista semiestruturada foi elaborado entre o período de 6 de dezembro de 2020 a 18 janeiro de 2021. As entrevistas serão realizadas a adolescentes com idade compreendida entre os 14 e os 19 anos, com um dos pais com qualquer tipo de cancro, em fase de diagnóstico e/ou tratamento. Previamente à consecução das entrevistas, solicitar-se-á a assinatura do consentimento informado aos adolescentes e aos seus pais, que consentirão a participação dos filhos no estudo.

Os dados colhidos de cada entrevista, serão transcritos e posteriormente avaliados de acordo com análise de conteúdo segundo o referencial de Bardin (2016).

Para a validação do guião de entrevista semiestruturada será efetuado um pré teste, de forma a aferir-se o instrumento e permitindo o treino do investigador.

O guião de entrevista cumpre todos os pressupostos éticos inerentes à consecução de estudos, tendo sido aprovado pela Comissão de Ética do Instituto Português de Oncologia de Coimbra (IPOC), Portugal, com o número de processo TI 25/2020.

\section{RESULTADOS E DISCUSSÃO}

O guião desenvolvido contempla questões que visam conhecer as experiências dos adolescentes relativamente ao coping familiar, à relação pai-filho, à sua avaliação sobre o cancro da mãe/do pai e aos seus processos de coping.

Para a exploração de cada variável foram elaboradas questões abertas que possibilitam ao adolescente expressar a sua vivência do cancro parental e explorar as suas necessidades. 


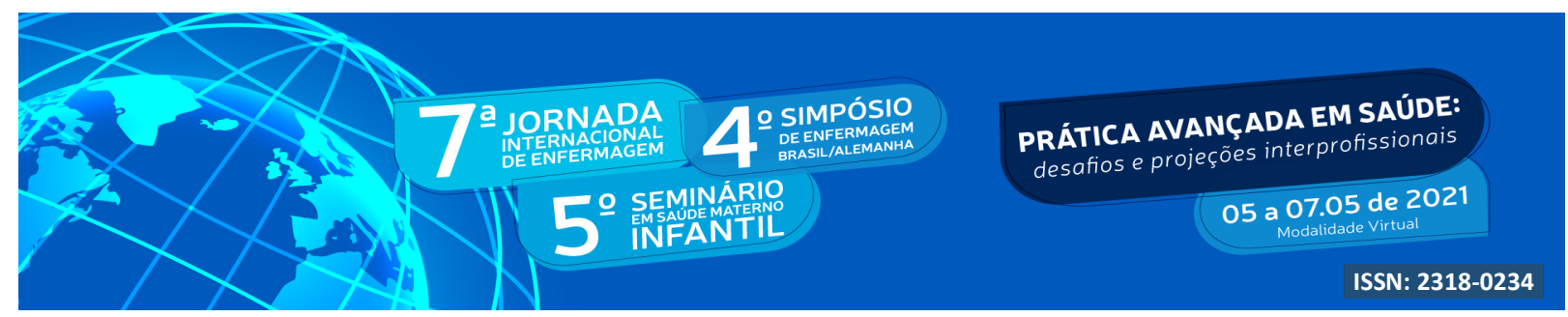

O guião de entrevista semiestruturada integra quatro blocos temáticos: I - Legitimação da Entrevista; II - Identificação geral; III - Abordagem da vivência do cancro parental e IV Questões finais e agradecimento.

O bloco temático III - Abordagem da vivência do cancro parental, tem como objetivo colher dados que permitam analisar a vivência do cancro parental pelo adolescente. As questões foram elaboradas com base nas variáveis moderadoras e mediadoras descritas no modelo de ajuste do adolescente ao cancro parental.

As variáveis moderadoras nos adolescentes incluem características como a idade, sexo, desenvolvimento cognitivo e socioemocional e experiências prévias de outro familiar próximo com cancro e/ou outra doença grave.

As variáveis mediadoras integram quatro conceitos fundamentais: coping familiar, relacionamento pais-filhos, avaliação do adolescente sobre o cancro dos pais e estratégias de coping.

A análise do discurso produzido pelos adolescentes nas entrevistas semiestruturadas, realizadas com base no modelo conceptual, permitirá avaliar o ajuste de cada adolescente ao cancro parental.

O ajuste do adolescente compreende o resultado a curto prazo da vivencia do cancro parental. E representa a performance do adolescente ao nível das dimensões emocional (alterações de humor e auto-estima), somática, social (desempenho escolar) e interpessoal. Um "mau ajuste" sugere que o adolescente apresenta mais problemas biopsicossociais do que a média da população, ao contrário o "bom ajuste", considera que os adolescentes filhos de pais com cancro têm o mesmo ou menor nível de problemas biopsicossociais que a média da população adolescente (Su \& Ryan-Wenger, 2007).

O ajuste do adolescente pode classificar-se em quatro dimensões: ansiedade, depressão, ajuste psicossocial e sintomas de resposta ao stress, e autoestima e performance académica (Su \& Ryan-Wenger, 2007). A classificação do ajuste do adolescente dependerá da análise do discurso, atendendo às suas respostas e às variáveis moderadoras e mediadoras.

A análise global das entrevistas permitirá avaliar e categorizar as necessidades dos adolescentes a vivenciar o cancro parental. 


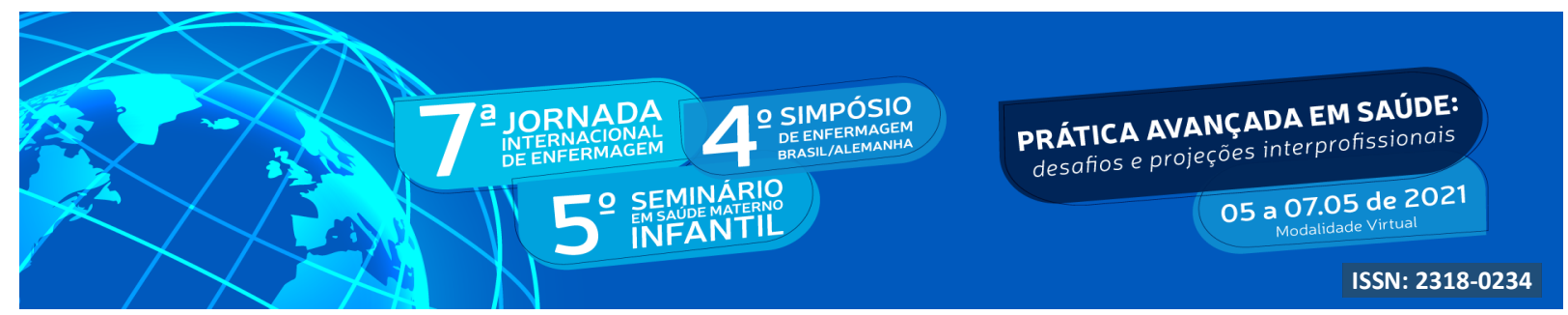

\section{CONCLUSÃO}

As entrevistas realizadas aos adolescentes, com recurso a um guião elaborado à luz do modelo conceptual do ajuste dos adolescentes ao cancro parental, permitirão o conhecimento e análise da vivência do cancro parental pelos adolescentes.

Os resultados das entrevistas permitirão analisar as necessidades dos adolescentes e serão uma base para a construção de um programa de intervenção de enfermagem para adolescentes e pais que vivenciam a experiência do cancro parental. De acordo com o modelo as intervenções de enfermagem autónomas devem considerar o impacto das variáveis moderadoras e mediadoras, por forma a promover o ajuste dos adolescentes ao cancro parental.

\section{REFERÊNCIAS}

ALEXANDER, E., O'CONNOR, M., RESS, C., \& HALKETT, G. (2019). A systematic review of the current interventions available to support children living with parental cancer. Patient Education and Counseling, 102, 1812-1821. Disponível em: https://www.sciencedirect.com/science/article/abs/pii/S0738399118308905?via\%3Dihub. doi: 10.1016/j.pec.2019.05.001

ARBER, A. (2016). How do Nurses "Think Family" and Support Parents Diagnosed with Cancer Who Have Dependent Children? Asian Oncology Nursing Society, 3, (214217). Disponível em: https://www.ncbi.nlm.nih.gov/pmc/articles/PMC5123514. doi: 10.4103/2347-5625.189809

AZARBARZIN, M., MALEKIAN, A., \& TALEGHANI, F. (2015). Effects of supportiveeducative program on quality of life of adolescents living with a parent with cancer. Iranian Journal of Nursing and Midwifery Research, 20 (557-581). Disponível em: http://www.ijnmrjournal.net/article.asp?issn=1735-

9066; year $=2015$; volume $=20$; issue $=5$; spage $=577$; epage $=581$; aulast $=$ Azarbarzin.$\quad$ doi: 10.4103/1735-9066.164510

BARBOSA, P. R. C. (2015). Adaptação da família com crianças/adolescentes ao diagnóstico de cancro parental (Dissertação de Mestrado). Instituto de Ciências Biomédicas Abel Salazar da Universidade do Porto (Medicina), Portugal. Disponível em: https://repositorioaberto.up.pt/bitstream/10216/90624/2/172184.pdf

BARDIN, L. Análise de Conteúdo. São Paulo: Edições 70. ISBN: 978-85-62938-04-7, 2016.

CHO, O.-H., YOO, Y.-S., \& HWANG, K.-H. (2015). Comparison of parent-child communication patterns and parental role satisfaction among mothers with and without breast cancer. Applied Nursing Research, 28(2), (163-168). Disponível em: https://pubmed.ncbi.nlm.nih.gov/25448055/. doi: 10.1016/j.apnr.2014.09.005. 


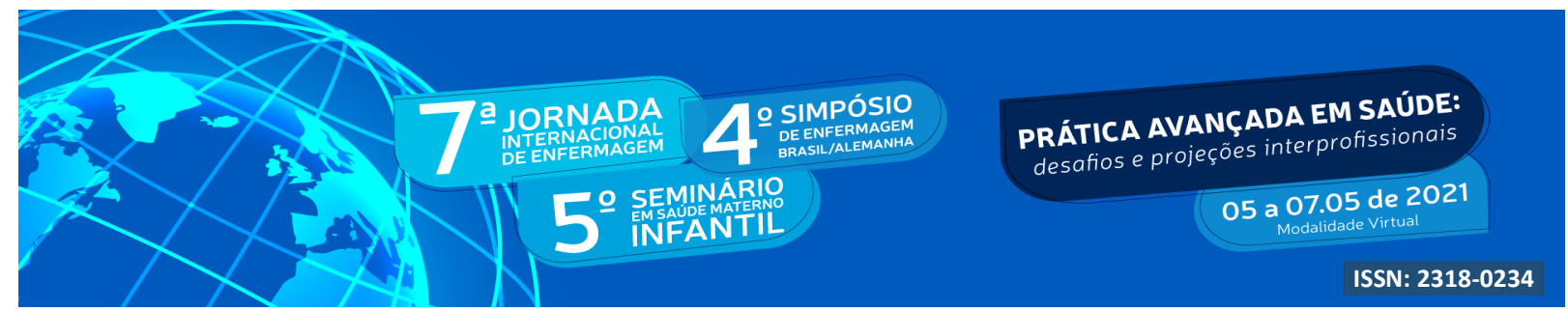

ELLIS, S.J., WAKEFIELD, C.E., ANTILL, G., BURNS, M., \& PATTERSON, P. (2016). Supporting children facing a parent's cancer diagnosis: a systematic review of children's psychosocial needs and existing interventions. European Journal of Cancer Care, (1-22). Disponível em: https://onlinelibrary.wiley.com/doi/epdf/10.1111/ecc.12432. doi: $10.1111 /$ ecc. 12432

GRABIAK, B. R.; BENDER, C. M., \& PUSKAR, K. R. (2007). The impact of parental cancer on the adolescent: An analysis of the literature. Pyscho-Oncology, 16 (127-137). Disponível em: https://pubmed.ncbi.nlm.nih.gov/16998950/. doi: 10.1002/pon.1083

INHESTERN, L., HALLER, A.-C., WLODARCZYK, O., \& BERGELT, C. (2016). Psychosocial interventions for families with parental cancer and barriers and facilitators to implementation and use - A systematic review. PloS One, 11(6), 1-20. doi: https://doi.org/10.1371/journal.pone.0156967

LEWIS, F., ZAHLIS, E., \& SHANDS, M. (2020). Upping my game as a parent: Attribued gains in participanting in a cancer parenting program for child-rearing parents with advanced cancer. Palliative and Supportive Care (1-7). Disponível em: https://pubmed.ncbi.nlm.nih.gov/32148212/. doi: 10.1017/S1478951520000103

O’NEILL, C., O’NEILL, C. S., \& SEMPLE, C. (2019). Children Navigation Parental Cancer: Outcomes of a Psychosocial Intervention. Comprehensive Child and Adolescent Nursing. (1-18). Disponível em: https://www.tandfonline.com/doi/full/10.1080/24694193.2019.1582727?scroll=top\&needA ccess $=$ true. doi: $10.1080 / 24694193.2019 .1582727$

OPPERMAN, C., \& CASSANDRA, K. (2001). Enfermagem Pediátrica Contemporânea. Loures: Lusociência.

ORDEM DOS ENFERMEIROS (2010). Regulamento das Competências Específicas do Enfermeiro Especialista em Saúde da Criança e do Jovem. Recuperado de: https://www.ordemenfermeiros.pt

PHILLIPS, F. (2014). Adolescents living with a parent with advanced cancer: a review of the literature. Psycho-Oncology, 23 (1323-1339). Disponível em: https://onlinelibrary.wiley.com/doi/full/10.1002/pon.3570. doi: 10.1002/pon.3570

SOCIEDADE PORTUGUESA DE ONCOLOGIA (2007). Falar Sobre Cancro. Volume 2. Disponível em: https://www.sponcologia.pt/fotos/editor2/publicacoes/vol.2 a pessoa_com_cancro.pdf

SHAH, B. K., ARMALY, J., \& SWIETER, E. (2017). Impact of Parental Cancer on Children. Anticancer Research, 37 (4025-4028). Disponível em: https://www.ncbi.nlm.nih.gov/pubmed/28739684. doi: 10.21873/anticanres.11787

SU, Y., \& RYAN-WENGER, N. (2007). Children's Adjustment to Parental Cancer. A theorical Model Development. Cancer Nursing, 30 (5) (362-381). Disponível em: https://journals.lww.com/cancernursingonline/Abstract/2007/09000/Children_s_Adjustment to Parental Cancer A.7.aspx. doi: 10.1097/01.NCC.0000290817.37442.

TAMURA, R., YAMAZAKI, T., \& UCHIBORI, M. (2021). "I'll Try My Best to be a Dad": The Experiences of Japanese Fathers with Cancer. Global Qualitative Research, 8 (1-15). Disponível em: https://us.sagepub.com/en-us/nam/open-access-at-sage. doi: $10.1177 / 2333393620975739$ journals.sagepub.com/home/gqn 